Publications of the Astronomical Society of the Pacific

100: 1582-1585, December 1988

\title{
ATMOSPHERIC REFRACTION EFFECTS ON THE NORRIS AND KECK MULTIOBJECT SPECTROGRAPHS
}

\author{
J. G. COHEN AND J. CROMER \\ Palomar Observatory, 105-24, California Institute of Technology, Pasadena, California 91125 \\ Received 1988 July 29, revised 1988 August 26
}

\begin{abstract}
Large field spectrographs are severely influenced by atmospheric refraction. We discuss the effect of differential refraction across the field as well as the chromatic effects for the Norris spectrograph under construction for use at Palomar Observatory and for a similar spectrograph for the Keck telescope on Mauna Kea.
\end{abstract}

Key words: instrumentation-spectroscopy-atmospheric refraction

\section{Introduction}

Atmospheric refraction affects multiobject spectrographs in two distinct ways. The first problem is the chromatic effect, which is due to the difference in the index of refraction of air as a function of wavelength. The image of a star is spread out into a line with fixed azimuth but varying altitude. In normal spectrographs this problem is always present, but it can be avoided by placing the slit at the parallactic angle (i.e., along the altitude axis). The appropriate formulae for this case are given in Fillipenko (1982). However, in multiobject spectrographs, even those that use multislits, the freedom to rotate the slit to any arbitrary orientation is often sacrificed for the optimization of coverage of high-priority objects in a given field. In multiobject spectrographs that use fiberoptic spectrograph feeds, the entrance aperture is round and, hence, no improvement in the situation by varying the spectrograph orientation is possible.

Under such circumstances the only way to correct for chromatic atmospheric refraction is the use of an atmospheric dispersion compensator (ADC). The usual ADC design is a pair of counter-rotating prisms as reviewed by Wallner and Wetherell (1980). Complex and expensive optics are required which may also introduce additional problems, i.e., ghost images due to reflections off the additional optical surfaces in the beam. Another design suggested by Richardson and Morbey (1988) involves a thick tiltable plate, but the correction, particularly for large wavelength ranges, is not as good. No ADC exists at present or is planned for the Hale telescope. Epps (1985) has designed an ADC for the Keck telescope.

The second effect introduced by the atmosphere is the differential refraction across the large field of a multiobject spectrograph. This problem is peculiar to large field instruments and is not present in normal spectrographs.

We have coded up calculations of both of these effects and discuss them in terms of their severity for the 5-m Hale telescope at Palomar and the 10-m Keck telescope on Mauna Kea. The code has been checked by reproducing the calculations of Bowen (1966) made for the Palomar Schmidt telescope with its $6^{\circ}$ field and those of Epps (1985) for the design of the ADC for the Keck 10-m telescope.

We have assumed that the Palomar site has a mean air pressure $(P)$ of $630 \mathrm{~mm}$ of $\mathrm{Hg}$, while that of Keck was taken as $456 \mathrm{~mm}$ of $\mathrm{Hg}$. The temperatures characteristic of each site were taken as $5^{\circ} \mathrm{C}$ for Palomar and $2^{\circ} \mathrm{C}$ for Keck, and the latitudes used are $33 \mathrm{~N}$ and $20 \mathrm{~N}$, respectively. Because to first order $(n-1)$ is proportional to $P$, the effect of atmospheric refraction will be reduced by a factor of 0.72 in the Keck situation as compared to the Palomar one. However, the mean seeing is better and, therefore, the constraints adopted for the Keck model are more severe.

The Norris spectrograph has fibers which are effectively 1.5 arc sec in diameter. We assume that any blur arising from atmospheric refraction effects which is larger than 0.5 arc sec (in effect, $1 / 3$ the diameter of the entrance slit) is not tolerable. For the Keck spectrograph the effective fiber diameter will be 1.0 arc sec and, therefore, we adopt 0.33 arc sec as our maximum tolerable blur. This means that, for any given situation, the effect of atmospheric dispersion on the Keck spectrograph is about the same as that on the Palomar spectrograph, because the lower atmospheric pressure is balanced by the better average seeing and the smaller fiber size adopted for the spectrograph feed.

\section{Chromatic Effects}

$(n-1)$ for air is a very strong function of wavelength in the near ultraviolet. Therefore, we have assumed that the Norris spectrograph lens will transmit over the wavelength range from $3800 \AA$ to $10000 \AA$ and that wave- 
lengths shorter that $3800 \AA$ will not be used. The chromatic dispersion spreads the image out in a line, whose length is a function of altitude. This is shown for Palomar in Figure 1, where the difference in position (in arc sec) of the monochromatic image from that at $3800 \AA$ is shown as a function of wavelength over the range in altitude from objects at the zenith (altitude $=90^{\circ}$ ) to those just above the horizon. The circles are $200 \AA$ apart. The same calculation for Mauna Kea is shown in Figure 2. If one ever managed to build a double spectrograph, as is eventually planned for Keck, then coverage over the full spectral range from $3200 \AA$ to $10000 \AA$ is feasible. The calculation of chromatic effects for Mauna Kea over the full spectral bandpass accessible from the ground with standard CCDs is given in Figure 3. Note that the effects are considerably worse.

We adopt the 0.5-arc-sec criterion for Palomar and the wavelength range over which the Norris lens has acceptable performance (i.e., $3800 \AA$ to $10000 \AA$ ). Figure 1 shows that only for objects which are less than $20^{\circ}$ from the zenith is the size of the image within our tolerances. Any object which is further from the zenith is blurred unacceptably and in an uncorrectable way. This mäy be

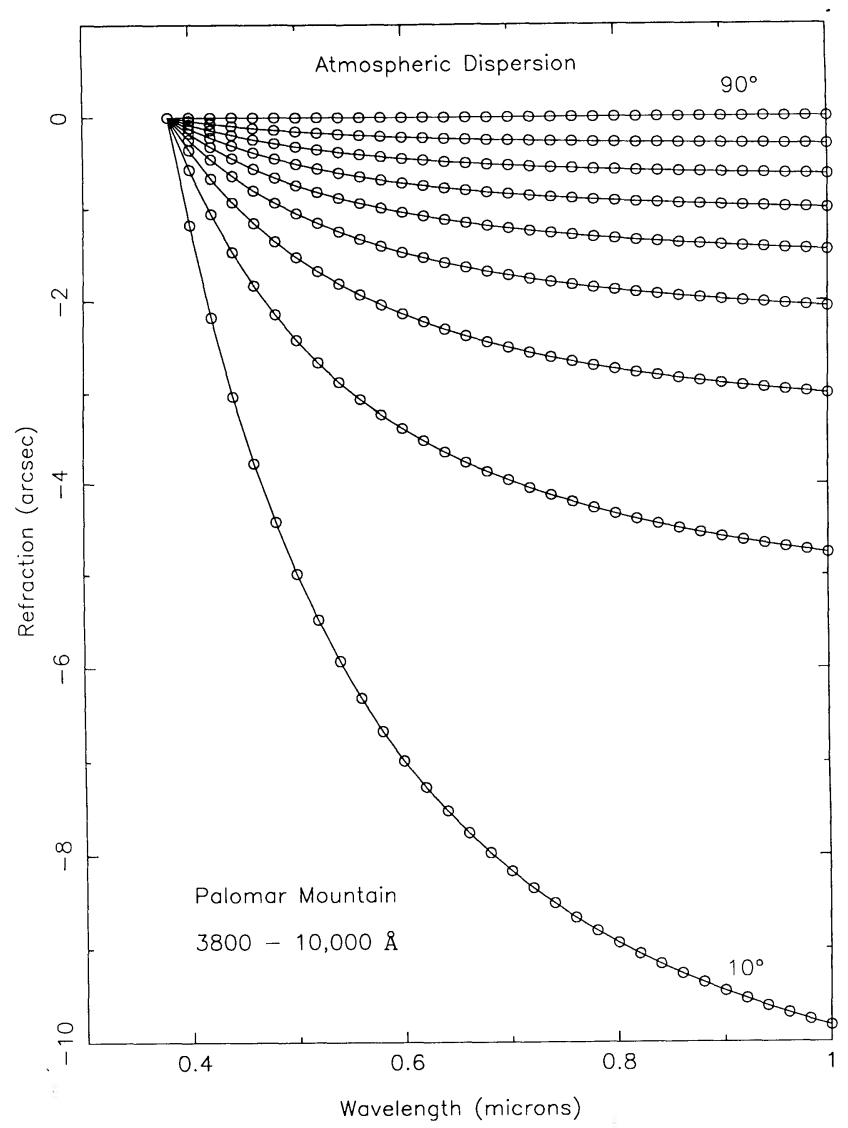

FIG. 1-The chromatic atmospheric dispersion in arc sec as a function of altitude for Palomar Mountain. The open circles are $200 \AA$ apart over the interval from $3800 \AA$ to $10000 \AA$. Image parameters for altitudes ranging from $90^{\circ}$ to $10^{\circ}$ in $10^{\circ}$ increments are shown.

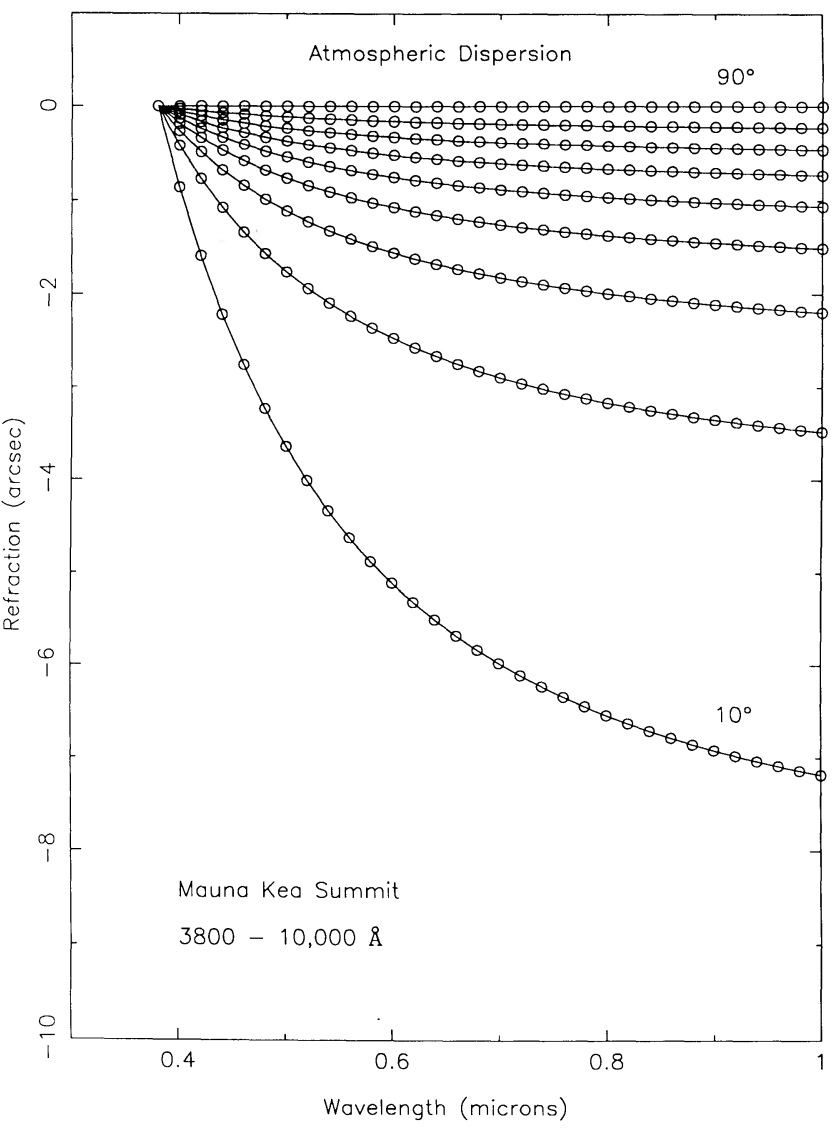

FIG. 2-The same as Figure 1 but for the summit of Mauna Kea.

tolerable for an instrument which has high spectral resolution, operates only over a small wavelength range, and is guided at the relevant wavelength. But for low-resolution spectrographs with broad spectral coverage, the consequences of atmospheric refraction are of serious concern. For example, at altitude $=30^{\circ}(\sec (z)=2.0)$, the image of a point source is almost 3 arc-sec long over the spectral range of the Norris spectrograph.

\section{Differential Effects Across the Field}

Because the amount of atmospheric refraction is a function of zenith distance, if the field becomes very large the differential atmospheric refraction across the field may become detectable. We consider a maximum exposure time of four hours across a field 20 arc min in diameter, the field of the Norris spectrograph. (The field of the Keck multifiber spectrograph will be 16 arc min.) We assume that the exposures begin not more than three hours east of the meridian. We assume that the guide star is at the center of the field and that all motion of the guide star is removed. Point sources are placed in a grid covering the area of the field, and their motion with respect to the guide star is calculated. This motion is a function of the duration of the exposure, the initial hour angle, the declination of the object, and the site parameters (i.e., $P$ and latitude and, to a minor extent, atmospheric tempera- 


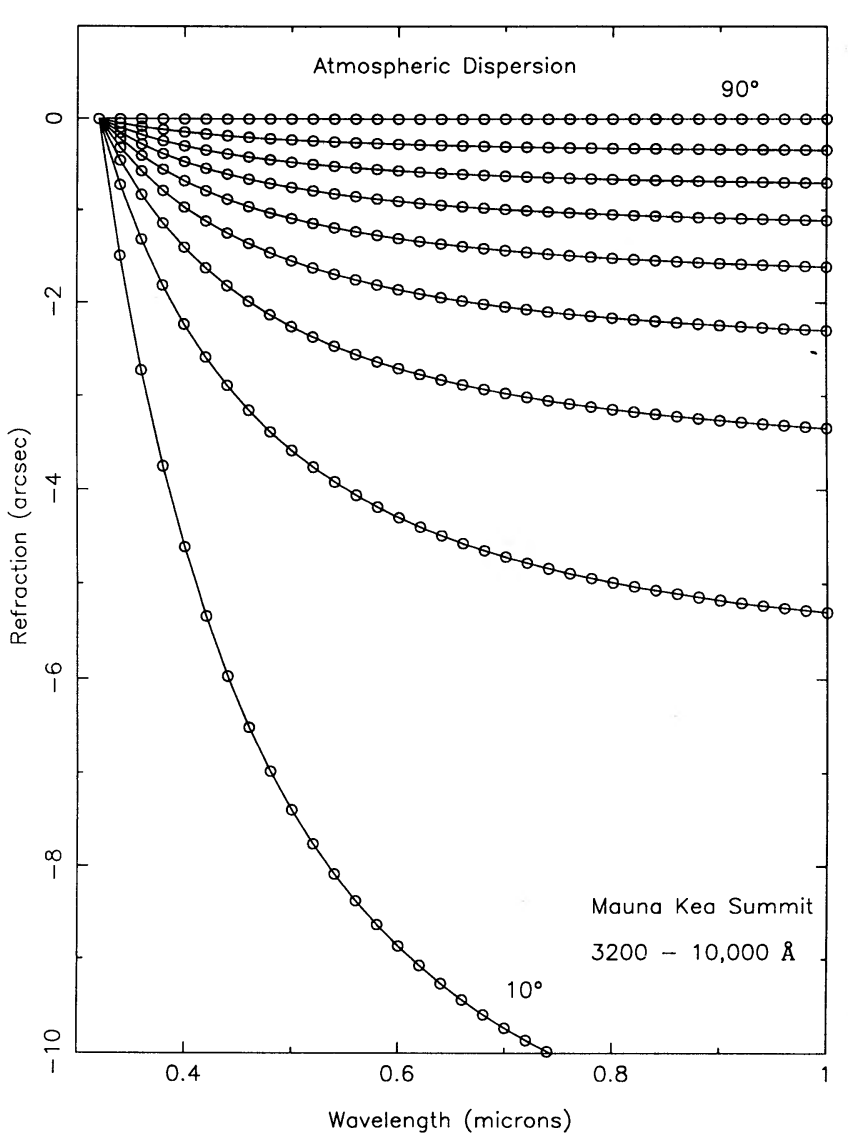

FIG. 3-The same as Figure 1 but for the summit of Mauna Kea and covering a wavelength range from $3200 \AA$ to $10000 \AA$.

ture). (While there is also a dependence on wavelength, in fact this is quite small and can be ignored.)

Figure 4 shows the image motion occurring during a four-hour exposure at $\delta=80^{\circ}$ beginning three hours east of the meridian for Palomar. The $y$ axis of the coordinate grid shown in the axes is the N-S axis, while the $x$ axis is E-W. Objects are placed at the location indicated by the scale on the figure, so that the full diameter of the field is 20 arc minutes. The motion of each object is shown at a magnified scale where one division (i.e., 1 arc min in position) is 1 arc sec of image motion. Note that in this case, which is characteristic of fields with $\delta$ north of the latitude of the site, the image motion appears to be approximated by a rotation about the center of the field. While the image motion is unacceptably large, introduction of a small rotation of the field would probably make it acceptable. This rotation can be introduced by rotating the fiber-optic feed about its mounting on the Cassegrain ring.

Figure 5 shows the situation for a four-hour exposure beginning three hours east of the meridian at a declination of $-20^{\circ}$ from Palomar. Again the image motions are intolerably large, but this time the image motion is a scale change (i.e., a radial motion) combined with a rotation of the field and will probably be unacceptably large even

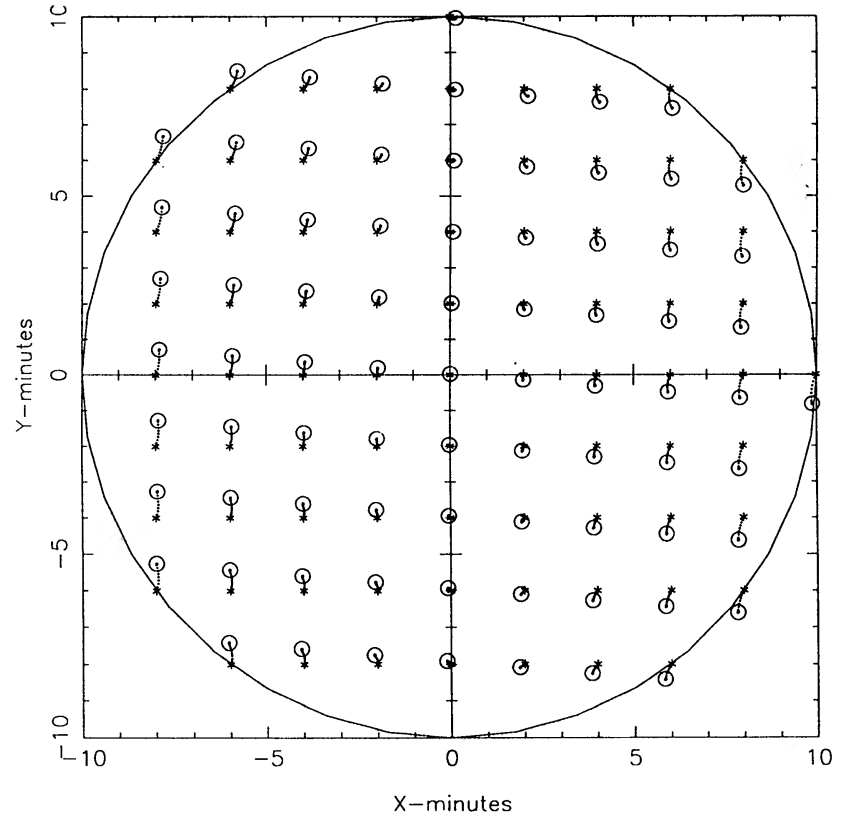

Fig. 4-Differential refraction across the field of the Norris spectrograph is shown for all exposure from Palomar Mountain at $\delta=80^{\circ}$ which is four hours long and begins with the field center three hours east of the meridian. The scale of the axes refers to the position of the points with respect to the center of the field, while the motion of the points is shown on an exaggerated scale in which 1 arc sec of motion corresponds to a tick mark (i.e., 1 arc sec of motion is equivalent to 1 arc min of position).

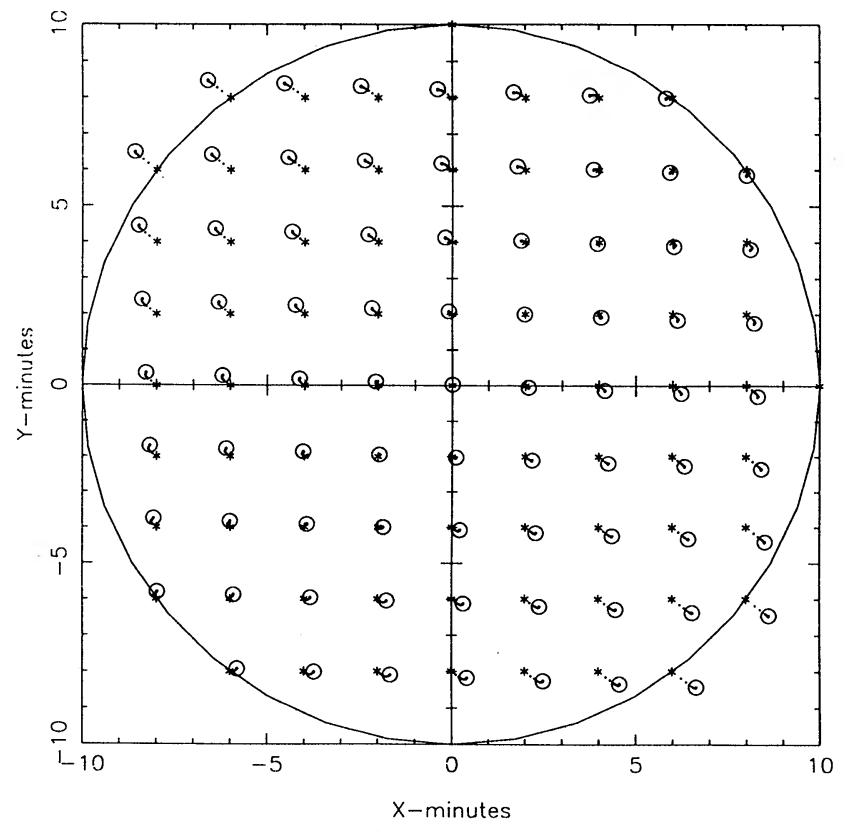

Fig. 5-The same as Figure 4 but for an exposure at $\delta=-20^{\circ}$.

after a rotation correction is applied.

The situation is summarized in Table I, which displays for the standard situation (four-hour exposure beginning three hours east of the meridian from Palomar) the maximum image motion of any point with respect to the 
TABLE I

Maximum Image Motion of a Point with Respect to Center of the Field

\begin{tabular}{cccc}
\hline $\begin{array}{c}\text { Dec } \\
\text { (degrees) }\end{array}$ & $\begin{array}{c}\text { Max. Motion } \\
\text { (arc-sec) }\end{array}$ & Characteristic form & Notes \\
\hline 80 & 0.7 & rotation \\
70 & 0.3 & rotation & \\
60 & 0.2 & & \\
50 & 0.15 & & \\
40 & 0.10 & & \\
30 & 0.10 & pincushion+rotation & \\
20 & 0.10 & & \\
10 & 0.15 & & 1 \\
0 & 0.30 & & 1 \\
-10 & 0.40 & & \\
-20 & 0.75 & & \\
-30 & 0.40 & & \\
-40 & 1.25 & & \\
\hline \hline
\end{tabular}

(Note: 1-3 hour exposure beginning 1.5 hours east of meridian)

central guide star as a function of declination.

We thus see that for Palomar there are two regions of concern where the image motion exceeds 0.5 arc sec, fields north of about $75^{\circ}$ and those south of $-15^{\circ}$. While rotation of the instrument on the Cassegrain mounting ring (Cass ring) during long exposures will reduce the problem in the northern fields to an acceptable level, it is only a partial solution to the image motion problems of the southern fields.

The necessary precision of rotational motion is perhaps 0.25 arc sec over a radius of 10 arc min, or $1 / 2400$ radians in the position angle of the ring. This corresponds to an accuracy of 1.4 arc min in the Cass ring position angle. This motion must be achievable during exposures, which should be possible if the instrument is properly balanced so that the Cass ring need not be locked into position, or if the lock release mechanism operates smoothly, so that it can be used during exposures. The present encoding and positioning scheme on the Hale telescope for rotation of the Cass ring is not adequate for such fine motions, but improvements are planned which should be completed before the Norris spectrograph becomes operational. The Keck instrument rotator should be able to provide the requisite fine control of position angle.

We are grateful to the Kenneth T. and Eileen L. Norris Foundation, whose generosity has provided the means to design and build the Norris spectrograph. We are grateful to Robert Brucato for helpful discussions.

\section{REFERENCES}

Bowen, I. S. 1966, Quart, J.R.A.S., 8, 22.

Epps, H. 1985, in The Design of the Keck Observatory and Telescope, ed. J. E. Nelson, T. S. Mast, and S. M. Faber, Keck Observatory Report, No. 90, p. 4.

Fillipenko, A. V. 1982, Pub. A.S.P., 94, 715.

Richardson, E. H., and Morbey, C. L. 1988, in Instrumentation for Ground Based Optical Astronomy, ed. L. B. Robinson (New York: Springer Verlag), p. 177.

Wallner, E. P., and Wetherell, W. B. 1980, in Optical and Infrared Telescopes for the 1990s, ed. A. Hewett (Tucson, AZ: Kitt Peak National Observatory), p. 717. 